(C) 2013 IEEE. Personal use of this material is permitted. Permission from IEEE must be obtained for all other users, including reprinting/republishing this materials for advertising or promotional purposes, creating new collective works for resale or redistribution to servers or lists, or reuse of any copyrighted components of this work in other works." 


\title{
Proton-Radiation Tolerance of Silicon and SU-8 as Structural Materials for High-Reliability MEMS
}

\author{
Tobias Bandi, João Polido-Gomes, Antonia Neels, Alex Dommann, Laurent Marchand, and Herbert R. Shea
}

\begin{abstract}
The susceptibility of single-crystal silicon and SU-8 resonators to proton-radiation induced degradation was investigated. Both materials are in widespread use for microsystems structures, thus the stability of the mechanical properties must be ensured over the full device lifecycle. Effects of spacerelevant proton doses were examined by monitoring minute changes in the Young's modulus and by structural investigations using high-resolution X-ray diffraction (HRXRD). Single crystal silicon resonators were exposed to $10 \mathrm{MeV}$ and $60 \mathrm{MeV}$ protons with doses up to $10^{13} \mathrm{~cm}^{-2}$. Even at the highest doses neither a change of the Young's modulus was observed nor did Xray diffraction indicate the formation of elevated concentrations of structural defects. The compatibility of SU-8 with inorbit radiation environments was investigated at fluences of $10^{10}-10^{12} \mathrm{~cm}^{-2}$ using protons with energies ranging from $10 \mathrm{MeV}$ to $200 \mathrm{MeV}$. Its elastic modulus changed by less than $5.5 \%$ at the highest doses.

[2013-0009]
\end{abstract}

Index Terms-Accelerated aging, material reliability, microelectromechanical systems (MEMS), radiation effects, silicon, SU-8, Young's modulus

\section{INTRODUCTION}

$\mathbf{M}$ ICRO-ELECTROMECHANICAL systems (MEMS) for applications in environments with high radiation levels must be fabricated from materials that are able to resist degradation by ionizing and displacement damage mechanisms. Ionizing damage causes the formation of excitations and electron-hole pairs, inducing charge trapping or altering chemical bonding structures. If the momentum transfer to the target atoms is sufficiently high, displacement damage occurs, leading to structural defects in the atomic lattice. Protective shielding of microsystems could be envisaged but

Manuscript received January 7, 2013; revised March 25, 2013; accepted March 27, 2013. Date of publication June 21, 2013; date of current version November 25, 2013. This work was supported in part by the Networking/Partnering Initiative of the European Space Agency under Contract No. 21872. Subject Editor G. Stemme.

T. Bandi is with the Centre Suisse d'Électronique et Microtechnique SA (CSEM SA), 2002 Neuchâtel, Switzerland and also with the École Polytechnique Fédérale de Lausanne (EPFL), 2002 Neuchâtel, Switzerland (e-mail: tobias.bandi@csem.ch).

J. Polido-Gomes and H. R. Shea are with the École Polytechnique Fédérale de Lausanne, 1015 Lausanne, Switzerland (e-mail: joao.polidogomes@gmail.com; herbert.shea@epfl.ch).

A. Neels and A. Dommann are with the Centre Suisse d'Électronique et Microtechnique SA (CSEM SA), 2002 Neuchâtel, Switzerland (e-mail: antonia.neels@csem.ch; alex.dommann@csem.ch).

L. Marchand is with the European Space Agency, Noordwijk, 2201 BB Noordwijk, Netherlands (e-mail: Laurent.Marchand@esa.int).

Color versions of one or more of the figures in this paper are available online at http://ieeexplore.ieee.org.

Digital Object Identifier 10.1109/JMEMS.2013.2262596 for radiation-sensitive systems this quickly becomes prohibitive in mass and size, outweighing the advantages of miniaturization. For a review on the radiation tolerance of MEMS devices we refer to [1] and references therein. Charge trapping in dielectrics has been identified as a major radiationinduced cause of failure. The accumulation of charges in insulating materials is a common reliability issue for many electrostatically operated MEMS that can also be induced by high electric fields. In both cases counteracting the effects of trapped charge is possible by following basic rules such as minimizing the volume of dielectrics in critical areas and by designing devices whose operation is insensitive to trapped charge [1]. This can significantly improve the reliability [2] but cannot eliminate material-specific limitations such as the degradation of the mechanical and electrical properties of the materials themselves. Appropriate material selection is therefore at the heart of engineering of reliable MEMS and is one of the main factors determining the performance and the failure modes of devices. However, fabrication process constraints and functionality requirements limit the choices. Possible candidate materials need to be evaluated in detail for their ability to perform under harsh environmental conditions.

There is no general threshold for the radiation tolerance of MEMS materials, as the radiation-induced damage will affect different parts of a MEMS device differently. The acceptable dose levels depend on the fabrication processes, the functional role of a material, and the influence it exerts on the performance of the device.

Single crystal silicon is widely used as a structural material in MEMS. It is highly suitable for microsystems due to its versatility in fabrication and the high mechanical and chemical stability it offers. Radiation-induced damage has been investigated extensively in regards of its electrical properties. While being largely immune to ionizing damage, displacement damage has been reported to change minority carrier lifetimes and concentrations in electronic components. For a review on this topic we refer to [3] and references therein. However, in MEMS devices where silicon is used as a structural material, the functionality of the system is much more sensitive to changes in Young's modulus. The elasticity directly influences the vibrational modes of resonant structures which are standard building elements in MEMS devices. Resonances are used, for instance, in sensors for measuring a variety of variables such as the acceleration, pressure, and angular rate, in actuators for driving devices such as micromirrors and in electronic 
components such as resonator-based ladder filters. The accuracy of these systems is dependent on the stability of the vibrational modes over time (and hence radiation dose).

In single crystal silicon, it has been previously reported that the resonance frequency of micromirrors had changed shortly after proton irradiation of $1 \mathrm{MeV}$ and $4.3 \mathrm{MeV}$ protons at $100 \mathrm{Mrad}$ total ionizing dose (TID) [4]. After 3 weeks the devices had recovered the resonance frequency prior to irradiation. This observation was explained by the introduction of defects which were mobile at room temperature and therefore annealed out after the irradiation. Surface-micromachined polysilicon resonator beams irradiated by gamma- and electron radiation have been investigated in [5], [6]. A decrease of the resonance frequency and an increase of resistance were observed. The results were attributed to a combination of displacement damage, injection annealing, and thermal spike effects. The effect of fast neutrons on the electromechanical properties of several materials used in microsystems has been studied and reported recently [7].

An interesting candidate for polymer MEMS is SU-8, which was first developed as a negative photoresist, but it has quickly been recognized as a promising structural material [8]. The large number of crosslinking points in the precursor molecules lead to high chemical and mechanical stability of the cured resin. The curing reaction of the epoxy is initiated by exposure to UV, gamma-rays, or proton radiation which allows the fabrication of very high-aspect ratios and 3D-structures [9], [10]. Due to its biocompatibility and mechanical elasticity it is a candidate for microfluidics, sensing and actuating applications [11], [12]. Radiation tolerance in polymer MEMS devices is often very limited and large changes in the elasticity have been reported [13]. The effect of neutron irradiation on the hardness of SU-8 coatings, i.e., the ability of the material to resist plastic deformation, was investigated in [14]. No significant change of hardness was observed in the coating after irradiation. To our knowledge, no studies of the influence of proton radiation on the mechanical properties of SU-8 MEMS devices have been conducted to date.

The extent to which a space system is exposed to radiation can be modeled over the full lifetime of the system, taking into account the trajectory and the radiation shielding. The total ionizing damage dose absorbed by a space system shielded by $4 \mathrm{~mm}$ of aluminum is on the order of $1 \mathrm{krad} / \mathrm{year}$ in low earth orbits and $10 \mathrm{krad} / \mathrm{year}$ in geostationary orbits [15]. The total dose level usually requested in the qualification testing of generic EEE parts is $100 \mathrm{krad}$ [16]. For comparison, the expected ionizing dose of a tracking detector in the LargeHadron-Collider at CERN is $1 \mathrm{Mrad}$, obtained after 1.5 years of operation, which is much higher than most space doses [17].

Protons were selected for this irradiation campaign because they are the most abundant ionic species of irradiation in space [18]. Their energy lies principally between $0.1 \mathrm{MeV}$ and $400 \mathrm{MeV}$. They are more difficult to shield than electrons and heavier ions. SRIM 2008 [19] simulations show that protons of $10 \mathrm{MeV}$ can penetrate $0.6 \mathrm{~mm}$ of aluminum. For MEMS material screening they offer the advantage of inflicting both ionization damage and displacement damage [1], [16].

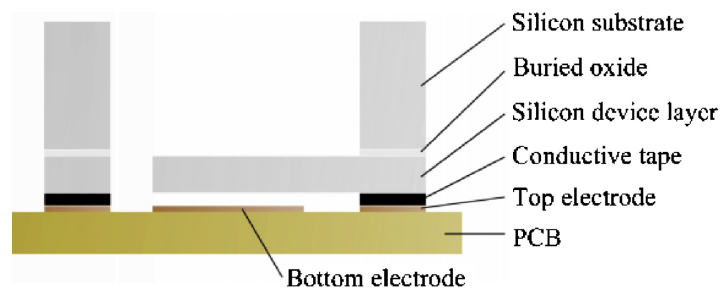

Fig. 1. Schematic cross-section of the silicon resonator chips. The devices were placed with the device layer on the PCB. Conductive tape was used to attach the devices and to electrically connect the device layer for electrostatic actuation of the resonators.

In Section II the fabrication of the MEMS devices and the experimental setup for the dynamical characterization of the resonators are described. This section also contains the test conditions for the irradiations. Based on the selected proton energies and fluences the resulting absorbed doses were calculated. The results of the structural and dynamical investigations after irradiation are reported in Section III. This section also contains a discussion of the observations, in the context of reported radiation effects in silicon and polymers.

\section{EXPERIMENTAL}

For the first harmonic mode of a cantilever, the resonance frequency $f$ is given by

$$
f=\frac{1.875^{2}}{2 \pi} * \frac{t}{l^{2}} \sqrt{\frac{E}{12 \rho}}
$$

where $t$ is the resonator thickness, $l$ is its length, $E$ is the Young's modulus, and $\rho$ is the density. The resonance frequency is very sensitive to changes in the material elasticity as $\Delta f / f=1 / 2 * \Delta E / E$. By monitoring the radiation-induced change of the resonance frequency the change in Young's modulus was calculated. This method is an elegant way to investigate mechanical properties of materials in structures whose geometry, dimensions, and fabrication processes are relevant and directly applicable to MEMS technology. For the purpose of isolating radiation-induced effects in the materials themselves, the device structures were designed such that no additional materials (dielectric, coils, etc.) were required for operation and characterization.

\section{A. Fabrication of Silicon Cantilevers}

Resonators (cantilevers) with lengths of $1.5 \mathrm{~mm}$ and $2 \mathrm{~mm}$, width $80 \mu \mathrm{m}$, and thickness $50 \mu \mathrm{m}$ were fabricated on $\mathrm{Si}(001)$ silicon-on-insulator (SOI) wafers. The chip substrate formed a frame enclosing an area of $1 \mathrm{~cm}^{2}$. The resonators were patterned into the device layer, pointing inward along the (110) crystal direction (Fig. 2(a)). Standard lithography and deep reactive ion etching were used to form the resonators.

Subsequently they were released by dry etching of the silicon substrate wafer from the backside followed by a hydrofluoric acid etching step to remove the buried oxide. As schematically depicted in Fig. 1 the chips were placed on PCB substrates for testing. The PCB plates were lined with copper electrodes for electrostatic actuation of the resonators. 
TABLE I

Dimensions AND AVERAge Resonance CHARACTERISTICS OF THE RESONATORS TESTED

\begin{tabular}{|c|c|c|c|c|c|c|c|}
\hline $\begin{array}{l}\text { Resonator } \\
\text { type }\end{array}$ & Material & $\begin{array}{l}\text { Length } \\
(\mu \mathrm{m})\end{array}$ & $\begin{array}{l}\text { Width } \\
(\mu \mathrm{m})\end{array}$ & $\begin{array}{l}\text { Thickness } \\
(\mu \mathrm{m})\end{array}$ & Clamping & $\begin{array}{l}\text { Resonance } \\
\text { frequency }(\mathrm{kHz})\end{array}$ & $\begin{array}{l}\text { Quality factor in } \\
\text { vacuum }\left(p \sim 10^{-3} \mathrm{mbar}\right)\end{array}$ \\
\hline Cantilever & $\mathrm{Si}$ & 1500 & 80 & 50 & Clamped-free & 24.6 & 900 \\
\hline Cantilever & $\mathrm{Si}$ & 2000 & 80 & 50 & Clamped-free & 14.8 & 4200 \\
\hline Paddle & $\mathrm{Si}$ & $\begin{array}{l}\text { Paddle area } 0.18 \mathrm{~mm}^{2} \\
\text { Arm length } 800 \mu \mathrm{m} \\
\text { Arm width } 80 \mu \mathrm{m} \\
\text { Arm thickness } 50 \mu \mathrm{m}\end{array}$ & & & & & \\
\hline Cantilever & SU-8 & 1000 & 120 & 100 & Clamped-free & 32.6 & 35 \\
\hline Cantilever & SU-8 & 800 & 120 & 100 & Clamped-free & 49.1 & 23 \\
\hline Cantilever & SU-8 & 1500 & 120 & 100 & Double clamped & 86.0 & 19 \\
\hline Cantilever & SU-8 & 1000 & 120 & 100 & Double clamped & 192.8 & 27 \\
\hline
\end{tabular}

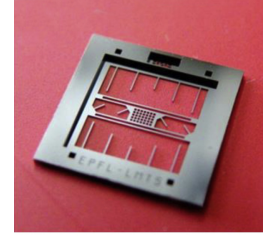

(a)

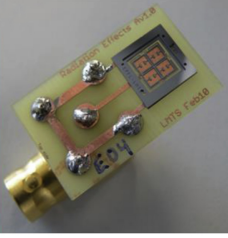

(b)

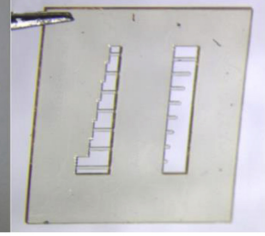

(c)
Fig. 2. (a) One type of single crystal silicon resonator chip (chip is $10 \mathrm{~mm}$ on a side, 14 cantilevers) and (b) different chips mounted on a PCB. The PCB contains copper lines for electrostatic actuation of the resonators. (c) SU-8 resonator chip. The size of the chip is $9 \mathrm{~mm} \times 9 \mathrm{~mm}$. Only the longest and second-longest SU-8 resonators were used for this research.

Table I summarizes the geometries of the resonators used. Figs. 2(a) and (b) show a single crystal silicon device chip and a silicon chip mounted on the PCB, respectively. In addition to the clamped-free cantilevers paddle-type resonators were also used. This geometry consisted of a rectangular tab suspended by a cantilever arm [as shown in Fig. 2(b)]. Despite the differences in the geometry always the same component of the elastic modulus tensor was measured because all resonators were only operated in the first out-of-plane mode.

\section{B. Fabrication of SU-8 Resonators}

The polymer devices were fabricated using commercial SU-8 (GM1075) obtained from Gersteltec. The fabrication process is schematically shown in Fig. 3. A sacrificial layer of dextran was deposited on a silicon handling wafer. The devices were formed by spinning of SU-8 precursor onto the handling wafer, soft-baking at $95{ }^{\circ} \mathrm{C}$, exposure to UV-light $\left(200 \mathrm{~mJ} / \mathrm{cm}^{2}\right)$, and subsequent post-baking at $120{ }^{\circ} \mathrm{C}$. This process was repeated twice to first form the device layer with the resonators and then the chip substrate. After this the unexposed SU-8 was dissolved in PGMEA (propylene glycol methyl ether acetate). The chips were released in de-ionized water and glued on a PCB for handling and testing. During the whole test campaign the SU-8 chips were not removed from the PCB support.

Piezoelectric elements obtained from NOLIAC A/S were used to actuate the SU-8 beams. The plate actuators (model CMAP 09) were $10 \mathrm{~mm}$ large, $10 \mathrm{~mm}$ wide and $2 \mathrm{~mm}$ thick. The tested resonators were $100 \mu \mathrm{m}$ wide and $120 \mu \mathrm{m}$ thick. Their lengths were $0.8 \mathrm{~mm}$ and $1.0 \mathrm{~mm}$ for the clamped-free

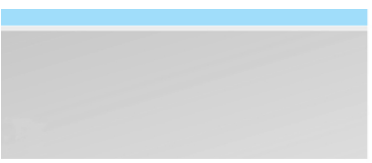

(a)

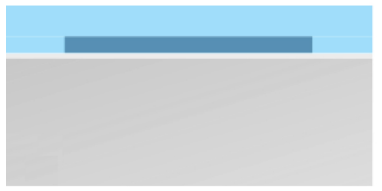

(c)

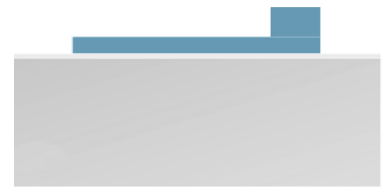

(e)

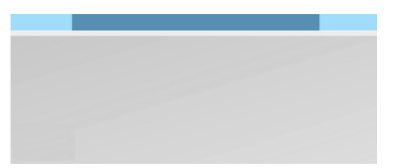

(b)

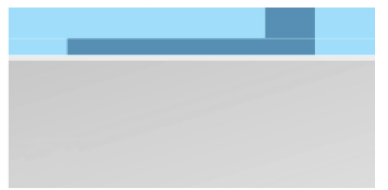

(d)

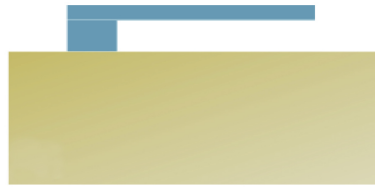

(f)
Fig. 3. Schematic representation of the fabrication process of the SU-8 devices. (a) Process started with a silicon wafer that served as a substrate for the fabrication (gray). Next a sacrificial layer of dextran was deposited and the spinning of the first layer of SU-8 took place (in light gray and blue, respectively). (b) After soft-baking the first SU-8 layer was exposed to the UV-light to define the resonators. Then the post-exposure bake (PEB) was made. The exposed region is shown in dark blue. (c) Spinning of the second layer of SU-8 on top of the first layer. (d) Soft-baking and selective exposure to UV-light was made to form the body of the chip. This was followed by the second PEB. (e) Unexposed SU-8 was then developed in PGMEA; the dextran layer was dissolved in DI-water to release the chip. (f) For testing the chips were attached to a PCB by carbon-loaded tape.

resonators and $1.0 \mathrm{~mm}$ and $1.5 \mathrm{~mm}$ for the clamped-clamped beams [Table I and Fig. 2(c)].

\section{Resonance Characterization}

The dynamical response of the resonators to actuation was determined by laser doppler vibrometry using a Polytec MVS400 instrument. A detailed description and characterization of the measurement setup has been reported previously [4]. During the resonance measurements the samples were placed inside a vacuum chamber $\left(p<3.5 \times 10^{-3}\right.$ mbar $)$ and the temperature was kept constant at $45 \pm 0.2{ }^{\circ} \mathrm{C}$ after a bake-out at 80 ${ }^{\circ} \mathrm{C}$. Constant temperatures throughout all measurements were used to suppress the effect of temperature on the resonance frequency. The bake-out served to minimize the effects of humidity variations over the duration of the test campaign. A frequency resolution of $0.5 \mathrm{~Hz}$ was used on the silicon 
TABLE II

IRRADIATION PARAMETERS FOR THE SILICON DEVICES

\begin{tabular}{ccccccc}
\hline Sample Proton energy & $\begin{array}{c}\text { Flux } \\
(\mathrm{MeV})\end{array}$ & $\begin{array}{c}\text { Fluence } \\
\left(\mathrm{cm}^{-2} \mathrm{~s}^{-1}\right)\end{array}$ & $\begin{array}{c}\text { TID } \\
\left(\mathrm{cm}^{-2}\right)\end{array}$ & $\begin{array}{c}\text { TDD } \\
(\mathrm{krad})\end{array}$ & $\begin{array}{c}\text { \# resonators } \\
\text { tested }\end{array}$ \\
\hline 1 & 10 & $10^{11}$ & $10^{13}$ & 5900 & 1500 & 7 \\
2 & 60 & $10^{9}$ & $10^{11}$ & 14 & 2 & 8 \\
3 & 60 & $10^{10}$ & $10^{12}$ & 140 & 17 & 4 \\
4 & 60 & $10^{11}$ & $10^{13}$ & 1400 & 167 & 7 \\
Control & - & - & - & - & - & 4 \\
\hline
\end{tabular}

resonators and $\leq 200 \mathrm{~Hz}$ on the SU-8 resonators. This was mainly limited by the width of the resonance curves. The standard deviation of the resonance frequency in un-irradiated resonators was determined by repeated measurements and was below $0.05 \%$ for the silicon resonators and $0.5 \%$ in the SU-8 resonators. Typical resonance frequencies and quality factors of the resonators are shown in Table I. The quality factor of the SU-8 resonators was comparable to values of SU-8 microresonators reported in the literature [20]. This indicates that the energy dissipation was dominated by the same mechanism as identified there, i.e., internal material damping. The double-clamped cantilevers possessed quality factors similar to the single-clamped resonators, suggesting that they were not under large internal tensile stresses, which would result in string-like oscillation characteristics and higher $Q$-factors [20]. The absence of buckling caused by excessive compressive internal stresses was confirmed by white-light interferometry (Wyko NT1100).

\section{Proton Irradiation Conditions}

SRIM 2008 [19], [21] was utilized to simulate the effect of proton irradiation on the materials and to calculate the absorbed doses. The TID was calculated from the ionizing (electronic) stopping power $\mathrm{d} E_{\mathrm{e}} / \mathrm{d} x$ :

$$
\operatorname{TID}[\mathrm{MeV} / g]=\frac{\mathrm{d} E_{e} / \mathrm{d} x}{\rho} * \Phi
$$

$\Phi$ is the particle fluence and $\rho$ is the density. The total displacement dose (TDD) was deduced based on the non-ionizing energy loss (NIEL) using the Kinchin-Pease relationship [22]:

$$
\operatorname{TDD}[\mathrm{MeV} / g]=\frac{M\left(\mathrm{~d} E_{n} / \mathrm{d} x\right)}{\rho} * \Phi
$$

where

$$
M=\frac{1}{1000}\left(\frac{T_{n}}{4}+2\right)
$$

$T_{\mathrm{n}}$ is the threshold energy for vacancy formation. In silicon $T_{\mathrm{n}}$ is $21 \mathrm{eV}$ [22]. For polymers it is difficult to estimate the exact displacement damage threshold as they contain more than one type of chemical bonds but it typically lies in the range of $10-30 \mathrm{eV}$ [23]. A value of $28 \mathrm{eV}$ was used for SU-8. These calculations served as a basis for the selection of the proton fluences.

The irradiations were carried out in two campaigns at the proton irradiation facility of the Paul-Scherrer Institute (Villigen, $\mathrm{CH}$ ). Each chip, containing several resonators, was irradiated under different proton radiation conditions.
TABLE III

IRRADIATION PARAMETERS OF THE SU-8 DEVICES

\begin{tabular}{ccccccc}
\hline Sample & $\begin{array}{c}\text { Proton energy } \\
(\mathrm{MeV})\end{array}$ & $\begin{array}{c}\text { Flux } \\
\left(\mathrm{cm}^{-2} \mathrm{~s}^{-1}\right)\end{array}$ & $\begin{array}{c}\text { Fluence } \\
\left(\mathrm{cm}^{-2}\right)\end{array}$ & $\begin{array}{c}\text { TID } \\
(\mathrm{krad})\end{array}$ & $\begin{array}{c}\text { TDD } \\
(\mathrm{rad})\end{array}$ & $\begin{array}{c}\text { \# resonators } \\
\text { tested }\end{array}$ \\
\hline 1 & 10 & $10^{8}$ & $10^{10}$ & 7 & 1.4 & 4 \\
2 & 10 & $10^{8}$ & $10^{11}$ & 74 & 14.1 & 3 \\
3 & 10 & $10^{8}$ & $10^{12}$ & 740 & 141 & 3 \\
4 & 19 & $10^{8}$ & $3 \times 10^{12}$ & 1360 & 228 & 3 \\
5 & 30 & $10^{8}$ & $10^{10}$ & 3 & 0.4 & 4 \\
6 & 30 & $10^{8}$ & $10^{11}$ & 29 & 3.8 & 4 \\
7 & 30 & $10^{8}$ & $10^{12}$ & 290 & 38.3 & 2 \\
8 & 30 & $10^{8}$ & $3 \times 10^{12}$ & 880 & 115 & 4 \\
9 & 60 & $10^{8}$ & $10^{10}$ & 2 & 0.2 & 4 \\
10 & 60 & $10^{8}$ & $10^{11}$ & 17 & 1.6 & 4 \\
11 & 60 & $10^{8}$ & $10^{12}$ & 170 & 15.7 & 3 \\
12 & 60 & $10^{8}$ & $3 \times 10^{12}$ & 500 & 472 & 3 \\
13 & 200 & $10^{8}$ & $10^{10}$ & 1 & 0.02 & 2 \\
14 & 200 & $10^{8}$ & $10^{12}$ & 70 & 1.8 & 2 \\
Control & - & - & - & - & - & 3 \\
\hline
\end{tabular}

Proton energies of $10 \mathrm{MeV}$ and $60 \mathrm{MeV}$ and fluences between $10^{11} \mathrm{~cm}^{-2}$ and $10^{13} \mathrm{~cm}^{-2}$ were used for the irradiation of the silicon resonators. The large fluences selected required an adaptation of the dose rate, which was between $10^{9} \mathrm{~cm}^{-2} \mathrm{~s}^{-1}$ and $10^{11} \mathrm{~cm}^{-2} \mathrm{~s}^{-1}$. Four chips were irradiated and 26 resonators were investigated in total. The activity of the samples abated to safe levels 3 months after proton irradiation, after which the post-irradiation measurements were carried out.

The SU-8 resonators were irradiated using protons of $10 \mathrm{MeV}, 19 \mathrm{MeV}, 30 \mathrm{MeV}, 60 \mathrm{MeV}$, and $200 \mathrm{MeV}$ and fluences between $10^{10} \mathrm{~cm}^{-2}$ and $3 \times 10^{12} \mathrm{~cm}^{-2}$. To prevent dose-rate dependent effects it was equal for all samples $\left(10^{8} \mathrm{~cm}^{-2} \mathrm{~s}^{-1}\right)$. The first measurement of the SU-8 resonators was made 2 weeks after irradiation, when the samples were released by the irradiation facility. Three months after the irradiation the dynamical characterization was repeated. Fourteen SU-8 chips were irradiated on which a total number of 45 resonators were tested. The irradiation conditions of the silicon and SU-8 devices are summarized in Tables II and III, respectively.

\section{RESULTS AND DISCUSSION}

\section{A. Single Crystal Silicon}

In the irradiated silicon no discoloration was observed. The results of the resonance characterization are summarized in Fig. 4, which shows the relative change in Young's modulus of the irradiated resonators.

Most of the resonators showed a $\Delta E / E$-value similar to the measurement uncertainty, but in four resonators a larger deviation was measured. Two of these resonators were irradiated at $140 \mathrm{krad}$ and two at $6 \mathrm{Mrad}$, but none at 1.4 Mrad. In both cases the resonators in question were not direct neighbors in the chip and the beam was much larger than the sample, thus a misalignment of the chip and the proton beam was ruled out as a possible reason for these observations. The deviation in the resonance frequency did not smoothly increase with the dose (notice the log-scale in Fig. 4) and the 


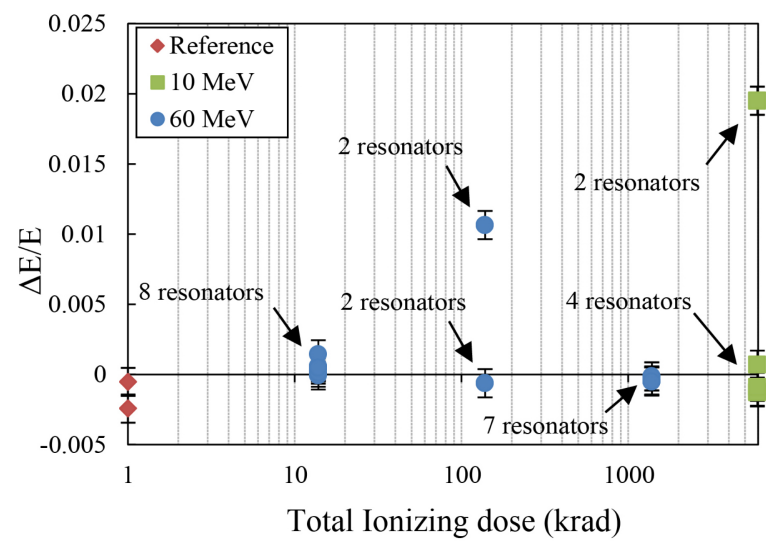

Fig. 4. Relative change in Young's modulus of the individual silicon cantilevers after $10 \mathrm{MeV}$ and $60 \mathrm{MeV}$ proton irradiation. For clarity the number of resonators is indicated where several measurement points overlap.

values in question were heavily displaced from the majority of data points. Therefore, these data points were probably rather related to experimental side effects such as airborne dust particles than to radiation-induced change of Young's modulus. The variation of the quality factor tended to increase with the total dose, however, the data were not conclusive on this and clearly further experiments must be made to clarify the effect of radiation on the resonator damping. As the quality factors of the silicon resonators were large, changes of the damping had only a negligible effect on the resonance frequency. These results suggest that the Young's modulus of single-crystal silicon was not significantly altered by proton fluences of up to $10^{13} \mathrm{~cm}^{-2}$ which corresponds to a dose of $5900 \mathrm{krad}$ TID for $10 \mathrm{MeV}$ protons.

It can be pointed out that the doses used in this study were about 100 times higher than typical space qualification levels and that possible degradation effects would be expected to be amplified accordingly.

The effect of proton irradiation on the crystal structure was investigated by high-resolution x-ray diffraction (HRXRD). The shape and width of the 004-Bragg peak were analyzed. These properties depend on the quality of the crystal, i.e., the concentration of structural defects such as dislocations, vacancies, and interstitials and on strain gradients. Fig. 5 shows the $\mathrm{Si}(004)$ diffraction peak before and after irradiation, both near the vicinity of the Bragg reflection and in a widerange scan (inset). Neither an increasing diffuse scattering, a peak broadening nor the appearance of a peak asymmetry indicating additional crystal defects or strain gradients were observed.

This indicated that the proton irradiation did not introduce significant amounts of stable lattice defects unlike heavier ion irradiations on which we had reported before: In [24] the effect of irradiation on single-crystal silicon resonant structures was investigated in devices irradiated by $5.5 \mathrm{MeV}$ alpha-particles (originating from an ${ }^{241} \mathrm{Am}$ source) to a total fluence of only approximately $8.7 \times 10^{10} \mathrm{~cm}^{-2}$. The diffuse scattering observed near the $\mathrm{Si}(224)$ reflection was attributed to displacement damage. The projected range of $5.5 \mathrm{MeV} \mathrm{He}{ }^{+}$of silicon is $27 \mu \mathrm{m}$ which is inside the device layer. The high stopping

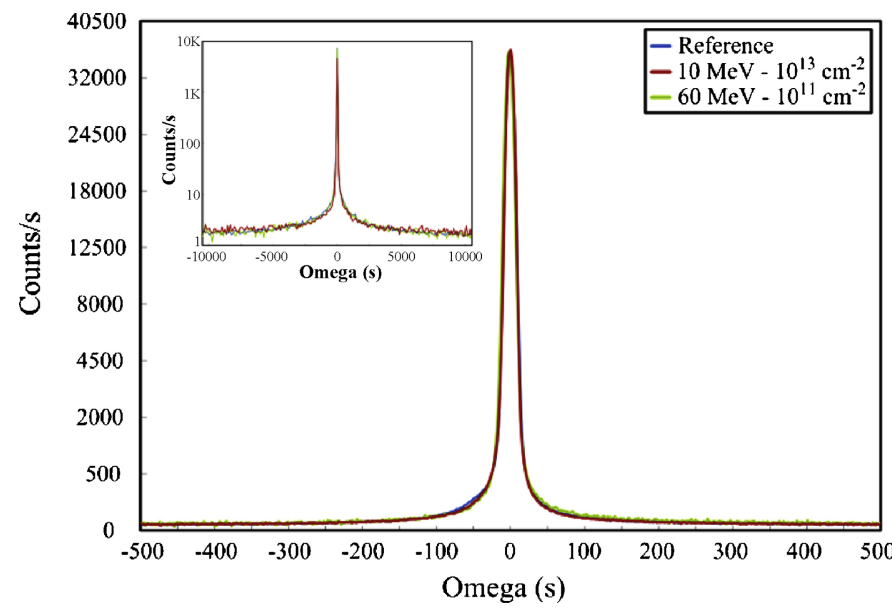

Fig. 5. HRXRD diffractogram of ( $\left.\begin{array}{lll}0 & 0 & 4\end{array}\right)$-reflection of the silicon samples. The graphs show rocking curve measurements ( $\omega$-scans) in the vicinity of the Bragg peak. The FWHM of the reflections is $0.0045^{\circ}$. (Inset) Wide $\omega$-scan using a larger step size. Within the experimental uncertainty no significant difference in the diffraction patterns was observed.

power at the end of the ion trajectory leads to higher defect concentrations and terminal subclusters [3] where larger and more stable defects are formed [34]. However, due to the large stopping power, these He-ions would not reach MEMS inside a spacecraft.

\section{B. $S U-8$}

No discolorations were observed after irradiation of the polymer samples. The changes in resonance frequency after irradiation were in the range of $-2.7 \%$ to $2.1 \%$ (maximum positive and negative change measured in any of the resonators). This corresponded to a variation of the Young's modulus between $-5.4 \%$ and $4.2 \%$.

In agreement with observations of the effect of irradiation on polymers reported in the literature [23], [25], the effect of radiation on SU-8 could not be simply related to the TID or to the total non-ionizing dose. The left graph in Fig. 6 shows the relative change in elasticity versus the proton fluence. No clear trend with regards to the particle fluence was observed (Left graph of Fig. 6). However, the evolution of the resonance frequency was found to be correlated with the proton energy and the electronic stopping power.

Fig. 6(right) shows the relative change in elasticity versus the proton energy. The data points with equal proton energy are the samples irradiated at different total proton fluences. At proton energies of $30 \mathrm{MeV}$ and higher a softening of the material was observed (negative $\Delta E / E$ ). At the lowest proton energies $(10 \mathrm{MeV}$ and $19 \mathrm{MeV})$ the Young's modulus was increased. An exception to this general observation was the sample irradiated at $10 \mathrm{MeV}$ and $10^{10} \mathrm{~cm}^{-12}$, where a softening was observed.

The threshold energy for the crossover from positive to negative $\Delta E / E$ was $20-25 \mathrm{MeV}$ which corresponded to a stopping power of approximately $3 \mathrm{eV} / \mathrm{nm}$. Although the quality factors of the SU-8 resonators were lower than in the silicon resonators, the variation of the quality factor did not significantly influence the resonance frequency shifts. 

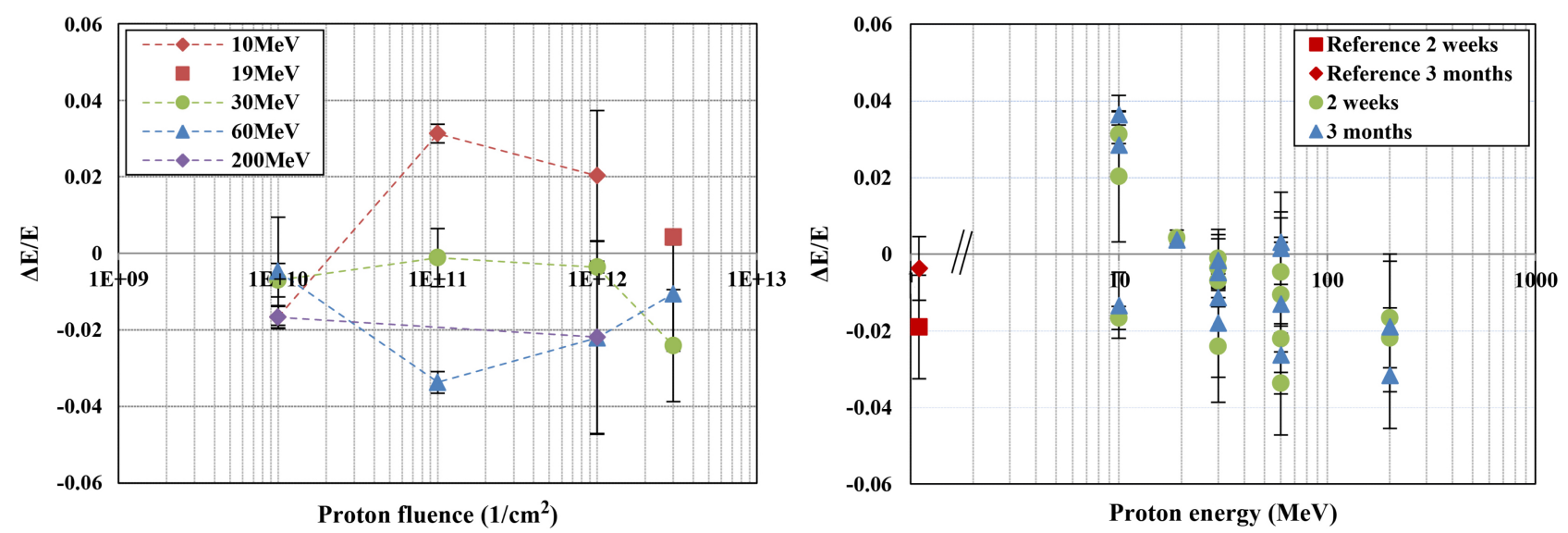

Fig. 6. Change in Young's modulus (average and standard deviation of all measured resonators on one chip) of SU-8 resonators after proton irradiation. The left graph shows the changes versus the proton fluence. The dashed lines are a guide for the eye. The right graph shows the change in elasticity versus the proton energy, $(\bullet) 2$ weeks after irradiation and (\) 3 months after irradiation. Points with equal proton energy are devices irradiated at different total fluences.

\section{Data From the SU-8 Control Samples}

On the proton-irradiated chips the average and standard deviation of the change in $\Delta E / E$ between the measurements 2 weeks and 3 months after the irradiation was $0.003 \pm 0.006$. However, on the control chip this difference was - 0.015 (see left graph of Fig. 6), which is larger than in any of the irradiated chips. This was caused by one of the three resonators on the control chip which showed large changes of the resonance frequency between the measurements. It is not possible to prove with certainty whether this was a genuine result or if it may have been caused by a dust particle lying on the resonator. However, it can be noted that this effect was transient and that the other resonators on the control chip were not showing such a behavior.

\section{Radiation-Induced Degradation in Polymers}

We briefly review radiation-induced degradation mechanisms in polymers and then discuss the observations on SU-8.

Changes in the structural properties of polymers are caused by ionization, radical formation, and atomic displacement. All of these processes can lead both to chain scissioning and crosslinking [23]. The chemical structure of the polymers determines the susceptibility to radiation damage and the dominance of specific radiation effects. For instance, aromatic compounds act as energy sinks due to the electronic delocalization. Transfer of energy from excited species to aromatic compounds thus effectively reduces the energy available for chemical damage [26]. The average SU-8 precursor molecule is based on four 'Bisphenol-A'-like subunits, each of which contains two aromatic rings. This may contribute to the good radiation tolerance of SU-8. Energy dissipation in the form of heat increases the mobility of active species and can influence the defect formation and annealing as well as the chemical reactions occurring. A significant heating effect during the irradiation of SU-8 was ruled out by analysis of the SRIM stopping power calculations: Under the (unlikely) assumption that all ionizing energy was transformed into thermal energy and that the full dose was applied instantaneously the change
TABLE IV

PARAMETERS OF Proton-IRRAdiation DAMAge IN SU-8: COLUMN 1 Contains the Electronic STOPPING POWER AND COLUMN 2 THE Nuclear Stopping Power of Protons IN SU-8. These Parameters WERE CALCULATED USING SRIM 2008.

\begin{tabular}{lcc}
\hline Radiation & $\begin{array}{c}\mathrm{d} E_{\mathrm{e}} / \mathrm{d} x \\
(\mathrm{eV} / \mathrm{nm})\end{array}$ & $\begin{array}{c}\mathrm{d} E_{\mathrm{n}} / \mathrm{d} x \\
(\mathrm{eV} / \mathrm{nm})\end{array}$ \\
\hline $10 \mathrm{MeV} \mathrm{H}^{+}$ & 5.6 & $1.0 \times 10^{-3}$ \\
$30 \mathrm{MeV} \mathrm{H}^{+}$ & 2.2 & $2.9 \times 10^{-4}$ \\
$60 \mathrm{MeV} \mathrm{H}^{+}$ & 1.3 & $1.2 \times 10^{-4}$ \\
$200 \mathrm{MeV} \mathrm{H}^{+}$ & 0.5 & $1.4 \times 10^{-5}$ \\
\hline
\end{tabular}

of temperature at the highest fluences was $19 \mathrm{~K}$ in SU-8 (80 ${ }^{\circ} \mathrm{C}$ in the silicon devices).

The degree of damage imparted by electronic and nuclear stopping, respectively, depends on the mass and energy of the impinging particles. Table IV shows the stopping powers of protons in SU-8, as determined from SRIM calculations. The highest vacancy formation rate due to the passing $\mathrm{H}^{+}$-ions and the recoil atoms occurred at $10 \mathrm{MeV}$ and was $\sim 10^{-5}$ vacancy $/ \mathrm{nm}$. Thus, although the nuclear displacement leads to chain disruptions and therefore can contribute to structural modifications, the effect of nuclear damage was very low in the present case and was assumed to be negligible.

Electronic stopping causes the formation of excited and ionized species. The susceptibility of polymers to irradiation has been reported to be dependent on the extent of electronic stopping power $\mathrm{d} E_{\mathrm{e}} / \mathrm{d} x$, i.e., the amount of energy converted to excitations and ionization per unit path length of the particle trajectory. Such observations are also referred to as 'LET effects' as the total stopping power $\mathrm{d} E / \mathrm{d} x$ is closely related to the linear energy transfer (LET). The LET describes the energy loss from the perspective of the particle, whereas the stopping power is a measure of the energy absorbed by the material. LET-dependent radiation effects, in which the observed damage is correlated to the electronic stopping power of the radiation, were investigated in various polymers 
including aromatic and aliphatic compounds [23], [25], [27], solid alanine [28], polystyrene, and polysilanes [29], [30]. However, [31] reported similar effects on the elongation to break in Ultem and Kapton irradiated by $3 \mathrm{MeV}$ protons, $2 \mathrm{MeV}$ electrons, or gamma-rays. Thresholds for the appearance of LET effects were also reported. In PMMA the critical LET value was found to be $15 \mathrm{eV} / \mathrm{nm}$. Below the threshold LET the scissioning efficiency was fairly constant. At higher stopping powers the scissioning efficiency was reduced [23], [25], which was related to elevated crosslinking efficiency due to higher ionization densities. LET thresholds were observed to be lower in aromatic polymers than in aliphatic compounds [27], [32].

LET effects have been explained by overlapping of ion tracks (inter-track effects) or by interactions between ionization events caused by the same ion (intra-track effects). The onset of track overlapping has been reported to occur at fluences of $10^{12} \mathrm{~cm}^{-2}$ to $10^{13} \mathrm{~cm}^{-2}$, depending on the particle LET [28], [33], [25]. As we already saw deviations of the Young's modulus at lower fluences, we assume that the observed change in elasticity was related to the concentration of ionizations within single tracks and the following scenario appears to be most realistic: At high energies, i.e., lower stopping powers, the ionizations were isolated events and chainscissioning dominated. Lower energy protons which deposited more energy in their track created higher concentrations of active species and favored crosslinking. The threshold for the dominance of one over the other process was found to be at proton energies of $20-25 \mathrm{MeV}$, corresponding to a stopping power of approximately $3 \mathrm{eV} / \mathrm{nm}$. This value is comparable to the findings of a previous study of poly(di- $n$ hexylsilane) where a threshold value of $10 \mathrm{eV} / \mathrm{nm}$ was reported [30]. Although this scenario qualitatively fits the experimental observations and is in agreement with concepts reported in the literature, several uncertainties remain. For instance, the change in Young's modulus was correlated with the proton energy but at a given energy no clear trend with the proton fluence was found. In addition, the observed effects were small $(|\Delta E / E| \leq 5.5 \%)$, not much larger than the experimental variance. Therefore more detailed investigations would be required to clarify the fundamental processes by which proton radiation interacts with $\mathrm{SU}-8$.

\section{CONCLUSION}

The susceptibility of the Young's modulus of single crystal silicon and SU-8 MEMS structures to proton radiation damage was systematically investigated. Microfabricated silicon resonators were irradiated with proton beams of energy $10 \mathrm{MeV}$ and $60 \mathrm{MeV}$ and doses up to $10^{13} \mathrm{~cm}^{-2}$. No change in the resonance frequency or Young's modulus was observed. Investigations using high-resolution X-ray diffraction methods did not indicate the presence of elevated levels of structural defects or strain gradients within the material. The results thus suggest that the elasticity of single crystal silicon is highly stable at proton radiation levels comparable to many years in most orbits around the Earth.
The Young's modulus of SU-8 was found to vary less than $\pm 5.5 \%$ at proton fluences of up to $3 \times 10^{12} \mathrm{~cm}^{-2}$. A correlation of the sign and degree of change in elasticity with the proton energy was observed. We put forward that this was related to the radiation stopping power and the energy which was deposited in the ion tracks. Further investigations are required to clarify the processes on the molecular level. Nevertheless, the SU-8 resonators remained intact and fully functional, showing that the polymer was highly tolerant to proton irradiation.

The measurement of the dynamical properties of microresonators is a powerful method for the investigation of material properties in structures relevant to microsystems technology. Our work showed that single crystal silicon and SU-8 are tolerant to high doses of proton radiation and are very well suited for MEMS in space applications. The selected geometry, fabrication process, and operation mode add to the relevance and allow for transferability of these results to other types of MEMS devices.

\section{ACKNOWLEDGMENT}

We thank H. Wojtek and Ilia Britvitch from the PaulScherrer institute for their support during the irradiation campaigns. We also thank T. Rohr and G. van Papendrecht from ESTEC (TEC-QTE) for very helpful discussions.

\section{REFERENCES}

[1] H. R. Shea, "Radiation sensitivity of microelectromechanical system devices," J. Micro/Nanolith. MEMS MOEMS, vol. 8, no. 3, p. 031303 , Jul.-Sep. 2009.

[2] S. S. McClure, L. D. Edmonds, R. Mihailovich, A. H. Johnston, P. Alonzo, J. DeNatale, J. Lehman, and C. Yui, "Radiation effects in microelectromechanical systems (MEMS): RF Relays," IEEE Trans. Nucl. Sci., vol. 49, no. 6, pp. 3197-3202, Dec. 2002.

[3] J. R. Srour, C. J. Marshall, and P. W. Marshall, "Review of displacement damage effects in silicon devices," IEEE Trans. Nucl. Sci., vol. 50, no. 3, pp. 653-670, Jun. 2003.

[4] J. Gomes and H. R. Shea, "Displacement damage effects in silicon MEMS at high proton doses," Proc. SPIE, vol. 7928, p. 79280G, 2011.

[5] L. Wang, J. Tang, and Q.-A. Huang, "Gamma irradiation effects on surface-micromachined polysilicon resonators," J. Microelectromech. Syst., vol. 20, no. 5, pp. 1071-1073, Oct. 2011.

[6] L. Wang, J. Tang, and Q.-A. Huang, "Gamma and electron beam irradiation effects on the resistance of micromachined polycrystalline silicon beams," Sens.Actuators A, Phys., vol. 177, pp. 99-104, Apr. 2012.

[7] P. Gkotsis, V. Kilchytska, C. Fragkiadakis, P.B. Kirby, J.-P. Raskin, and L. A. Francis, "Effects of fast neutrons on the electromechanical properties of materials used in microsystems," J. Microelectromech. Syst., vol. 21, no. 6, pp. 1471-1483, Dec. 2012.

[8] H. Lorenz, M. Despont, N. Fahrni, J. Brugger, P. Vettiger, and P. Renaud, "High-aspect-ratio, ultrathick, negative-tone near-UV photoresist and its application for MEMS," Sens. Actuators A, Phys., vol. 64, no. 1, pp. 33-39, Jan. 1998.

[9] A. del Campo and C. Greiner, "SU-8: a photoresist for high-aspect-ratio and 3D submicron lithography," J. Micromech. Microeng., vol. 17, no. 6, pp. R81-R95, Jun. 2007.

[10] Y. Gonin, F. Munnik F. Benninger, F. Dias, and S. Mikhailov, "Comparison of a new photoresist (DiaPlate 133) with SU-8 using both X-ray and ion beam lithographies," J. Vac. Sci. Technol. B, vol. 22, no. 4, pp. 1982-1986, 2004

[11] L. J. Guerin, M. Bossel, M. Demierre, S. Calmes, and Ph. Renaud, "Simple and low cost fabrication of embedded microchannels by using a new thick-film photoplastic," in Proc. Transducers, vols. 1-2, 1997, pp. 1419-1422. 
[12] I. Roch, Ph. Bidaud, D. Collard, and L. Buchaillot, "Fabrication and characterization of an SU-8 gripper actuated by a shape memory alloy thin film," J. Micromech. Microeng., vol. 13, no. 2, pp. 330-336, Mar. 2003.

[13] M. Niklaus, S. Rosset, H. Shea, "Array of lenses with individually tunable focal-length based on transparent ion-implanted EAPs," in Proc. SPIE, vol. 7642, 2010, p. 76422K.

[14] M. J. Key, V. Cindro, and M. Lozano, "On the radiation tolerance of SU-8, a new material for gaseous microstructure radiation detector fabrication," Radiat. Phys. Chem., vol. 71, no. 5, pp. 1003-1007, Dec. 2004.

[15] A. L Hartzell, M. G. daSilva, and H. R. Shea, MEMS reliability (MEMS Reference Shelf Series), Boston, MA: Springer U.S., 2011, sec. 4.4.1.

[16] ESCC Basic Specification No. 22900. (2007) Total dose steady-state irradiation test method [Online]. (3). Available: https://escies.org

[17] H. Spieler, "Introduction to radiation-resistant semiconductor devices and circuits," AIP Conf. Proc. 7th Beam Instrumentation Workshop, 1997, vol. 390, pp. 23-49.

[18] E. G. Stassinopoulos and J. P. Raymond, "The space radiation environment for electronics," Proc. IEEE, vol. 76, no. 11, pp. 1423-1442, Nov. 1988.

[19] J. F. Ziegler, J. P. Biersack, and U. Littmark, Stopping and Range of Ions in Solids, New York: Pergamon Press, 1985.

[20] S. Schmid and C. Hierold, "Damping mechanisms of single-clamped and prestressed double-clamped resonant polymer microbeams," J. Appl. Phys., vol. 104, no. 9, p. 093516, 2008.

[21] J. F. Ziegler and J. M. Manoyan, "The stopping of ions in compounds," Nucl. Instrum. Methods Phys. Res. B, vol. 35, no. 3/4, pp. 215-228, Dec. 1988.

[22] S. R. Messenger, E. A. Burke, G. P. Summers, M. A. Xapsos, R. J. Walters, E. M. Jackson, and B. D. Weaver, "Nonionizing energy loss (NIEL) for heavy ions," IEEE Trans. Nucl. Sci., vol. 46, no. 6, pp. 1595-1602, Dec. 1999.

[23] E. H. Lee, "Ion-beam modification of polymeric materials - fundamental principles and applications," Nucl. Instrum. Methods B, vol. 151, no. 1-4, pp. 29-41, May 1999.

[24] A. Neels, G. Bourban, H. R. Shea, A. Schifferle, E. Mazza, and A. Dommann, "Aging of MEMS - Correlation of mechanical and structural properties," Procedia Chem., vol. 1, no. 1, pp. 820-823, Sep. 2009.

[25] E. H. Lee, G. R. Rao, and L. K. Mansur, "Hardness enhancement and crosslinking mechanisms in polystyrene irradiated with high energy ionbeams," Mater. Sci. Forum, vol. 248-249, pp. 135-146, 1997.

[26] A. Chapiro, "Chemical modifications in irradiated polymers," Nucl. Instrum. Methods B, vol. 32, no. 1-4, pp. 111-114, May 1988.

[27] T. Sasuga, H. Kudoh, and T. Seguchi, "High energy ion irradiation effects on polymer materials - Changes in mechanical properties of PE, PSF and PES," Polymer, vol. 40, no. 18, pp. 5095-5102, Aug. 1999.

[28] H. Koizumi, T. Ichikawa, H. Yoshida, H. Shibata, S. Tagawa, and Y. Yoshida, "Radical formation in the radiolysis of solid alanine by protons and helium ions," Nucl. Instrum. Methods B, vol. 117, no. 3, pp. 269-274, Sep. 1996

[29] S. Seki, S. Tsukuda, K. Maeda, Y. Matsui, A. Saeki, and S. Tagawa, "Inhomogeneous distribution of crosslinks in ion tracks in polystyrene and polysilanes," Phys. Rev. B, vol. 70, no. 14, p. 144203, Oct. 2004.

[30] S. Seki, K. Maeda, Y. Kumini, S. Tagawa, Y. Yoshida, H. Kudoh, M. Sugimoto, Y. Morita, T. Seguchi, T. Iwai, H. Shibata, K. Asai, and K. Ishigure, "Ion beam induced crosslinking reactions in poly(di-n-hexylsilane)," J. Phys. Chem. B, vol. 103, pp. 3034-3048, 1999.

[31] D. J. T. Hill and J. L. Hopewell, "Effects of $3 \mathrm{MeV}$ proton irradiation on the mechanical properties of polyimide films," Radiat. Phys. Chem., vol. 48, pp. 553-537, 1996.

[32] T. Sasuga, S. Kawanishi, T. Seguchi, and I. Kohno, "Proton irradiation effects on several organic polymers," Polymer, vol. 30, no. 11, pp. 2054-2059, Nov. 1989.
[33] A. Licciardello and O. Puglisi, "Anomalous molecular weight distribution in ion irradiated polystyrene near the gel transition," Nucl. Instrum. Methods B, vol. 91, no. 1-4, pp. 436-441, Jun. 1994.

[34] Z. F. Di, M. Q. Huang, Y. Q. Wang, and M. Nastasi, "Dynamic annealing versus thermal annealing effects on the formation of hydrogeninduced defects in silicon," Appl. Phys. Lett., vol. 97, no. 19, p. 194101, 2010

Tobias Bandi received the B.Sc. and M.Sc. degrees in nanoscience with major in physics from the University of Basel in 2007 and 2009, respectively.

$\mathrm{He}$ is currently conducting a PhD-thesis at CSEM SA in Neuchâtel $(\mathrm{CH})$ in the X-ray applications/Quality control laboratory and at EPFL Lausanne in the Microsystems for Space Technologies Laboratory (LMTS) of Prof. H.R. Shea. His research interests are related to radiation damage in MEMS materials and devices as well as microsystems reliability investigations by high-resolution $\mathrm{X}$-ray diffraction and in situ microscale mechanical testing.

João Polido-Gomes obtained a PhD degree in the field of MEMS reliability from the Open University, U.K. In his Post-Doctoral work at the Centre for Microsystems and Photonics, University of Strathclyde, he worked as a research fellow toward the design, testing, and characterization of MOEMS for beam steering scanning applications. In the summer of 2009, he joined Prof. Herbert Shea's group EPFL-LMTS as a Post-Doctoral assistant, working on reliability and radiation effects of silicon MEMS for space applications. His current research interests include design, testing and reliability, fabrication and development of MEMS for earth-bound and space applications.

Antonia Neels is heading the XRD Application Lab of CSEM's Microsystems Technology Division. She received her PhD degree in chemistry in 1995 from the University of Neuchâtel in Switzerland and the Diploma from the Humboldt University in Berlin, Germany in 1991. Her interest is focalized on the study of strain and defect mobility in silicon based materials and devices, reliability issues and the study of the relationship of micro- and nano-crystalline structures and macroscopic properties in material sciences and microsystems technology. She teaches applied crystallography at different Swiss universities and has published more than 170 academic research papers in the merging fields of chemistry, biology, physics, and microtechnology.

Alex Dommann is CTO of the CSEM, Centre Suisse d'Electronique et de Microtechnique S.A. at Neuchâtel. Together with Nico de Rooji, he has been heading the new Microsystems Technology division at CSEM since 2008. $\mathrm{He}$ received his $\mathrm{PhD}$ degree in solid state physics in 1988 from ETHZ in Switzerland.

His research concentrates on the structuring, coating, and characterization of thin films and MEMS structures. He is a member of different national and international committees and teaches MEMS technology and crystallography at different Swiss universities and has published more than 100 papers in the fields of thin films, MEMS reliability, and microtechnology.

Laurent Marchand is heading the Component Technology Section at ESA/ESTEC, the technical center of the European Space Agency and he is coordinating MNT working groups within ESA as well as in collaboration with the European space industry.

Herbert R. Shea (SM'2009) holds a B.Sc. degree (1991) in physics from McGill University, and a Ph.D. degree (1997) in physics from Harvard University. He developed carbon nanotube FETs at IBM's T.J. Watson Research Center from 1997 to 1999, then joined Lucent Technologies' Bell Labs in Murray Hill, NJ, where he became the technical manager of the Microsystems Technology group, specializing in MEMS reliability. In 2004 he founded the Microsystems for Space Technologies Laboratory at the EPFL (Ecole Polytechnique Fédérale de Lausanne) in Switzerland, where he has been an associate professor since 2011. Current research topics include micromachined soft polymer actuators and sensors, MEMS ion sources for micro-propulsion of small spacecraft, and MEMS reliability. 